\title{
Etapas en la evaluación de diferentes cefalosporinas: Un flujograma lógico
}

\author{
ERNA CONA T.*
}

\section{STAGES IN THE EVALUATION OF SEVERAL CEPHALOSPORINS: A LOGICAL ALGORITHM}

The selection of most appropriate cephalosporins to be tested by the microbiology laboratory constitutes a collaborative decision of the laboratory together with the infectious diseases specialist, the pharmacy, the medical staff, and the infection control committee. The considerations for this selection must include: to be approved by FDA, reliability of in vitro test, approved clinical efficacy, type of microorganism, the infection site, location of the patient (out versus in patient), to consider NCCLS recommendations and to minimize the emergence of resistance. According to these variables primary and second line schedules may be constructed.

Key words: Cephalosporins, Susceptibility test, Selection of antimicrobial agents.

Los cefalosporinas son uno de los grupos de antimicrobianos más importantes y numerosos dentro de los $\beta$ lactámicos, con formulaciones de uso oral y parenteral.

Clínicamente se los ha clasificado en base a su desarrollo histórico, propiedades estructurales comunes y espectro antimicrobiano, en cuatro generaciones.

Desde el punto de vista microbiológico, las cefalosporinas de primera generación muestran buena actividad frente a cocos Gram positivos como Staphylococcus aureus sensible a cloxacilina, Streptococcus pyogenes, Streptococcus pneumoniae y enterobacterias no productoras de $\beta$ lactamasas (cromosomales y/o plasmidiales).
Las cefalosporinas de segunda generación poseen gran actividad frente a Haemophilus influenzae, Moraxella catarrhalis, Neisserias patógenas, mayor actividad frente a enterobacterias que las de primera generación, pero son menos activas frente a $S$. aureus.

Las cefalosporinas de tercera generación constituyen uno de los grupos de antimicrobianos de mayor uso en la actualidad poseen gran actividad frente a bacilos Gram negativos tipo enterobacterias (excepto sobre cepas productoras de cefalosporinasas y/o $\beta$ lactamasas de espectro extendido), H. influenzae, Neisseria spp, S. pyogenes, S. pneumoniae, agregándose además en algunas de ellas acción frente a Pseudomonas aeruginosa (ceftazidima y

\footnotetext{
* Laboratorio de Microbiología, Hospital Clínico General Dr. Raúl Yazigi J., Clínica Indisa.
} 
cefoperazona). Por otra parte, exhiben una pobre o nula acción frente a $S$. aureus.

Las cefalosporinas de cuarta generación, muestran potente actividad frente a bacilos Gram negativos resistentes a cefalosporinas de tercera generación y cepas productoras de $\beta$ lactamasas de espectro extendido (BLEE).

La selección de la cefalosporina más apropiada para probar e informar en un antibiograma debe formar parte de una decisión conjunta entre el laboratorio de microbiología, infectólogo, farmacia, equipo médico y de control de infecciones intrahospitalarias.

Las consideraciones previas a la inclusión de un antimicrobiano en el antibiograma incluyen:

- Eficacia clínica probada según tipo de microorganismo y localización de la infección.

- Fármaco aprobado por la FDA (E.U.A.).

- Conocimiento de los patrones de susceptibilidad local.

- Costos.

- Minimizar la emergencia de resistencia.

- Propósitos de control de IIH.

- Considerar las sugerencias vigentes de NCCLS.

A lo anterior se suma la confiabilidad de los estudios de susceptibilidad in vitro en el laboratorio, con sus respectivos controles de calidad.

\section{Consideraciones en la selección de una cefalosporina según tipo de microorganismo}

\section{Enterobacteriaceas}

- Cefalotina: permite inferir susceptibilidad a cefalosporina de primera generación (cefradina, cefadroxilo), excepto cefazolina que se debe evaluar en forma individual.

- Cefuroxima y otras cefalosporinas segunda generación: evalúe en forma individual.

- Cefotaxima o ceftriaxona o ceftizoxima: son equivalentes en susceptibilidad.

- Cefixime: no aplicable en Morganella sp.

- Cefprozil: no aplicable en Providencia sp.

- Las cepas productoras de BLEE se deben informar como resistentes a todas las penicilinas, cefalosporinas y aztreonam.

Enterobacter, Citrobacter y Serratia spp pueden desarrollar resistencia intra tratamiento con cefalosporinas de tercera generación, por lo tanto, se debe reestudiar aislamientos repetidos.

Salmonella y Shigella spp: no informe cefalosporinas de primera y segunda generación como susceptibles.

\section{Pseudomonas spp y Acinetobacter spp}

Se recomienda evaluar sólo cefalosporinas de tercera, cuarta generación y asociación de cefalosporinas con inhibidores de $\beta$ lactamasa.

\section{Staphylococcus spp}

Debe utilizarse sensidisco de oxacilina de $1 \mu \mathrm{g}$. Si el halo de inhibición es $<13 \mathrm{~mm}$ se considera resistente y se informa resistencia a todos los $\beta$ lactámicos disponibles. No se recomienda evaluar cefalosporinas, combinaciones con inhibidores de $\beta$ lactamasas ni carbapenem en estos casos.

\section{Enterococcus spp}

Se debe tener precaución ya que las cefalosporinas pueden aparecer activas in vitro, pero no son efectivas clínicamente; por lo tanto, no deben ser evaluados, ni menos ser informados como sensibles.

\section{Haemophilus spp}

En cepas procedentes de procesos invasores sólo evalúe cefalosporinas de tercera generación. Las cefalosporinas de segunda y tercera generación oral pueden usarse como terapia empírica en infección respiratoria, los estudios de susceptibilidad no resultan útiles en estos casos. Sólo tendría utilidad con fines de vigilancia epidemiológica.

Las cepas no productoras de $\beta$ lactamasa y resistentes a ampicilina deben ser consideradas resistentes a todas las combinaciones con inhibidores de $\beta$ lactamasas y cefalosporinas de segunda generación, a pesar de que aparentemente resulten susceptibles in vitro.

\section{Streptococcus pneumoniae}

Se debe emplear sensidisco de oxacilina de $1 \mu \mathrm{g}$. Si el halo de inhibición es $>20 \mathrm{~mm}$ se considera susceptible a todas las penicilinas y cefalosporinas. 
Tabla 1. Selección de cefalosporinas para antibiograma según microorganismo y muestra

\begin{tabular}{|c|c|}
\hline \multicolumn{2}{|c|}{ Enterobacterias en orina } \\
\hline Ambulatorio & $\begin{array}{l}\text { Primario } \\
\text { cefalotina (cefradina-cefadroxilo) } \\
\text { Complementario } \\
\text { cefuroxima } \\
\text { ceftriaxona o cefotaxima } \\
\text { (cefixima oral) }\end{array}$ \\
\hline Hospitalizado & $\begin{array}{l}\text { Primario } \\
\text { cefalotina } \\
\text { cefazolina } \\
\text { cefuroxima } \\
\text { cefotaxima o ceftriaxona } \\
\text { Complementario } \\
\text { ceftazidima } \\
\text { cefoperazona } \\
\text { cefepime }\end{array}$ \\
\hline
\end{tabular}

Enterobacterias en otras localizaciones

$\begin{array}{cl}\text { Ambulatorio } & \begin{array}{c}\text { Primario } \\ \text { cefalotina } \\ \text { Complementario } \\ \text { cefuroxima } \\ \text { cefotaxima o ceftriaxiona }\end{array} \\ \text { Hospitalizado } & \begin{array}{l}\text { Primario } \\ \text { cefalotina } \\ \text { cefuroxima }\end{array}\end{array}$

cefotaxima o ceftriaxona

Complementario

cefoperazona

ceftazidima

cefoperazona/sulbactam

cefepime

\section{Enterobacterias enteropatógenas}

Salmonella sp extraintestinal, evalúe cefalosporinas de 3a. generación

\section{Pseudomonas spp procedentes de secreciones y orina}

\begin{tabular}{|c|c|}
\hline Ambulatorio & $\begin{array}{l}\text { Primario } \\
\text { cefotaxima } \\
\text { ceftazidima } \\
\text { Complementario } \\
\text { cefoperazona } \\
\text { cefoperazona/sulbactam } \\
\text { cefepime }\end{array}$ \\
\hline Hospitalizado & $\begin{array}{l}\text { Primario } \\
\text { ceftazidima } \\
\text { cefoperazona } \\
\text { Complementario } \\
\text { cefoperazona/sulbactam } \\
\text { cefepime }\end{array}$ \\
\hline
\end{tabular}

Si es $<19 \mathrm{~mm}$, será preciso determinar susceptibilidad a penicilina y cefalosporinas de segunda, tercera y cuarta generación por algún método de CIM confiable. En cepas obtenidas de procesos invasores siempre determine la CIM.

La mejor selección de una cefalosporina para un antibiograma debe considerar además del tipo de microorganismo, el sitio anatómico de aislamiento y la condición del paciente (ambulatorio u hospitalizado). De acuerdo a estas variables se pueden recomendar esquemas primarios y complementarios en caso de presentar resistencia (Tabla 1).

\section{Neisseria gonorrhoeae}

Se recomienda evaluar ceftriaxona.

\section{Neisseria meningitidis}

Los métodos de susceptibilidad por difusión no son confiables, debe enviarse la cepa a centro de referencia.

\section{Haemophilus spp de origen respiratorio}

Se recomienda evaluar cefuroxima sólo si la cepa es resistente a ampicilina. 
Tabla 2. Cefalosporinas disponibles en el mercado chileno

\author{
Cefalosporinas de 1ra. generación \\ - cefadroxilo \\ - cefalexina \\ - cefazolina \\ - cefradina
}

Cefalosporinas de 2da. generación

- cefaclor

- cefprozil

- cefuroxima

Cefalosporinas de 3ra. generación

- cefixime

- cefoperazona

- cefotaxima

- ceftazidima

- ceftriaxona

Cefalosporinas 3ra. generación/inhibidor

de $\beta$ lactamasa

- Cefoperazona/sulbactam

Cefalosporinas de 4ta. generación

- Cefepime

\section{RESUMEN}

La selección de la cefalosporina más apropiada para testear e informar por el laboratorio de microbiología, forma parte de una decisión conjunta entre el laboratorio, infectólogo, farmacia, staff médico y comité de control de infecciones.
Las consideraciones en la selección incluyen: la aprobación del fármaco por la FDA, confiabilidad aceptable del test in vitro, eficacia clínica probada, tipo de microorganismo, sitio anatómico del aislamiento la localización del paciente (ambulatorio u hospitalizado), considerar las sugerencias vigentes del NCCLS, junto con minimizar la emergencia de resistencia. De acuerdo a estas variables se pueden recomendar esquemas primarios y complementarios.

\section{BIBLIOGRAFÍA}

1.- NCCLS. Performance Standards for Antimicrobial Susceptibility Testing Volume 21 Number 1 January 2001.

2.- MELLA S, ZEMELMAN C, BELLO H et al. Propiedades microbiológicas, clasificación y relación estructura-actividad de cefalosporinas e importancia de las cefalosporinas de cuarta generación. Rev Chil Infect (2001); (1): 7-19.

3.-ZEMELMAN R, NORAMBUENA R, VERGARA L et al. Los antibióticos $\beta$ lactámicos: agrupación según su estructura química y sus propiedades bacteriológicas. Rev Méd Chile 1987; 115: 983-91.

4.- MARSHALL W F, BLAIR J E. The cephalosporins. Mayo Clinic Proc 1999; 74: 187-95.

5.- PAYA E. Ventajas y desventajas de las cefalosporinas orales de segunda y tercera generación. Rev Chil Infect 1997; 14 (4): 233-8.

6.-ZEMELMANN R, BELLO H, DOMÍNGUEZ $M$ et al. Activity of imipenem, third-generation cephalosporins, aztreonam, and ciprofloxacin against multi-resistant Gram negative bacilli isolated from Chilean hospitals. J Antimicrob Chemother 1993; 32: 413-9.

Correspondencia a:

Erna Cona Trujillo

E-mail: econa@ctcinternet.cl 